\title{
BMJ Global Health Perspectives of host faculty and trainees on international visiting faculty to paediatric academic departments in East Africa
}

\author{
Christiana M Russ, ${ }^{1}$ Lakshmi Ganapathi, ${ }^{2}$ Diana Marangu, ${ }^{3}$ Melanie Silverman, ${ }^{1}$ \\ Edward Kija, ${ }^{4}$ Sabrina Bakeera-Kitaka, ${ }^{5}$ Ahmed Laving ${ }^{3}$
}

To cite: Russ CM, Ganapathi L, Marangu D, et al. Perspectives of host faculty and trainees on international visiting faculty to paediatric academic departments in East Africa. BMJ Global Health 2016;1: e000097. doi:10.1136/ bmjgh-2016-000097

- Additional material is available. To view please visit the journal (http://dx.doi.org/ 10.1136/bmjgh-2016000097)

Received 24 May 2016 Revised 14 September 2016 Accepted 15 September 2016

CrossMark

For numbered affiliations see end of article.

\section{Correspondence to}

Dr Christiana M Russ;

Christiana.Russ@childrens.

harvard.edu

\section{ABSTRACT \\ Background: Investments in faculty exchanges to build physician workforce capacity are increasing. Little attention has been paid to the expectations of host institution faculty and trainees. This prospective qualitative research study explored faculty and resident perspectives about guest faculty in paediatric departments in East Africa, asking (1) What are the benefits and challenges of hosting guest faculty, (2) What factors influence the effectiveness of faculty visits and (3) How do host institutions prepare for faculty visits?}

Methods: We recruited 36 faculty members and residents from among four paediatric departments in East Africa to participate in semistructured interviews which were audio recorded and transcribed. Data were qualitatively analysed using principles of open coding and thematic analysis. We achieved saturation of themes. Results: Benefits of faculty visits varied based on the size and needs of host institutions. Emergent themes included the importance of guest faculty time commitment, and mutual preparation to ensure that visit goals and scheduling met host needs. We documented conflicts that developed around guest emotional responses and ethical approaches to clinical resource limitations, which some hosts tried to prepare for and mitigate. Imbalance in resources led to power differentials; some hosts sought partnerships to reestablish control over the process of having guests.

Conclusions: We identified that guest faculty can assist paediatric institutions in building capacity; however, effective visits require: (1) mutually agreed on goals with appropriate scheduling, visit length and commitment to ensure that the visits meet the host's needs, (2) careful selection and preparation of guest faculty to meet the host's goals, (3) emotional preparation by prospective guests along with host orientation to clinical work in the host's setting and (4) attention to funding sources for the visit and mitigation of resulting power differentials.

\section{BACKGROUND}

In countries suffering from high levels of poverty, capacity for training healthcare

\section{Key questions}

What is already known about this topic?

- Existing studies describe academic institution partnerships such as the Medical Education Partnership Initiative, focus primarily on undergraduate medical education or research, and note North-South power imbalances and a need for faculty development.

- Results from two small qualitative studies about guest faculty carried out in single institutions indicated that hosts have positive feelings about guest faculty, though concerns include cultural differences, poorly aligned expectations, need for more mutual partnership and ethics of guest faculty clinical decisions.

What are the new findings?

- This study explored host perspectives of guest faculty anonymously, in detail and at multiple sites in East Africa with interviews carried out by an East African physician.

- Hosts shared experiences of benefits and challenges with stories that highlighted sources of conflict, the need for mutuality and partnership in planning, careful guest selection and preparation particularly for working clinically with limited resources, and attention to power imbalances and resources.

\section{Recommendations for policy}

- Guest faculty visits may be more effective with the following: (1) mutually agreed on goals with appropriate scheduling, visit length and commitment to ensure that the visits meet the host's needs, (2) careful selection and preparation of guest faculty to meet the host's goals, (3) emotional preparation by prospective guests along with host orientation to clinical work in the host's setting, (4) attention to funding sources for the visit and mitigation of resulting power differentials.

- Formal partnerships may improve mutuality of faculty exchanges, mitigate inherent power imbalances and allow for more sustained, trusting and open relationships. 
workers is often limited by numbers of existing faculty and subspecialty expertise. The Lancet Commission on Education of Health Professionals for the 21st Century called for sharing and strengthening educational resources, and linking educational institutions worldwide. ${ }^{1}$ Many medical education programmes such as Rwanda's Human Resources for Health and the Peace Corp's Seed Global Health rely on guest faculty to enhance training opportunities. ${ }^{2-4}$

Given the dearth of paediatricians in many countries, multiple global networks collaborate to improve paediatric education, and several academic paediatric centres in high and low-income countries are developing partnerships. ${ }^{5-8}$ We studied new paediatric residency programmes in low-income countries and found common early reliance on guest faculty with both positive and negative attributes. ${ }^{9}$

Host perspectives of guest faculty are not well represented in the literature. A qualitative study of nine host physicians at one institution noted themes of (1) culture, context and concern, (2) expectations, intentions and miscommunications, and (3) partnership and the desire to share and gain knowledge. ${ }^{10}$ A survey of surgical and anaesthesia trainees in Uganda found that while the majority agreed that visiting faculty improved their training, many reported a neutral or negative impact on patient care and discomfort with the ethics of visiting faculty's clinical decisions. ${ }^{11}$

Most studies about global partnerships focus on undergraduate medical education, with common elements of success including ownership of the agenda by African partners, frequent communication between partners and a focus on developing local expertise onsite at the African institution. ${ }^{12}{ }^{13}$ In specialty fields such as paediatrics, the literature is composed primarily of project descriptions. ${ }^{14-17}$ There are emerging competencies in global health for professionals that are pertinent to, but not specific for, the roles of guest faculty. ${ }^{18-21}$

We report a prospective qualitative study in medical education designed to explore the perspectives of faculty and residents at academic institutions in East Africa regarding guest faculty. The study was guided by three key research questions: (1) What are the benefits and challenges of hosting guest faculty? (2) What are the major factors or guest faculty behaviours that influence the efficacy of guest faculty teaching? and (3) How do host institutions prepare for guest faculty visits.

\section{METHODS}

This research used a grounded theory approach to explore guest faculty visits, employing a stratified purposeful sampling strategy. We recruited academic centres in East Africa by emailing all paediatric chairpersons in three countries-Uganda, Tanzania and Kenya. These countries were selected given their use of English in medical training and existence of multiple faculty exchanges. Of the six programmes contacted, four agreed to participate. Programmes ranged in size from 2 to 80 paediatric 'registrars' or residents, with faculty sizes varying from 8 to 35 . The programmes had been in existence between 26 and 92 years.

We identified three subgroups: senior faculty with leadership roles, junior faculty and paediatric residents. We aimed to interview three individuals in each subgroup at each institution for a total of 36 participants. Research logistics required that we establish the sample in advance; we felt that 36 participants would allow for a fully saturated theory. We developed loosely structured sets of questions, reflecting the differing roles of faculty and residents, to guide exploration of different aspects of guest faculty visits (see online supplementary appendix 1).

The sole interviewer (DM) is a Kenyan physician working towards her $\mathrm{PhD}$, who was recruited separately as a paid research assistant. She recruited potential participants by email and at departmental meetings. All 36 in-depth, semistructured interviews were conducted between October and December 2014. Interviews were audio recorded and transcribed. In order to promote more candid discussions, we kept participant and institution names anonymous and removed identifying information after transcription.

Data were qualitatively analysed with the assistance of OpenCode V.4.01 software, ${ }^{\mathrm{i}}$ using principles of open coding and thematic analysis to identify broad patterns in the collected data. Modified axial coding using hierarchy coding maps was used in order to identify and clarify relationships between themes and to explore causal relationships. Three individuals separately coded the interviews and met to compare and resolve any differences. Multiple coding and cross-checking were used in the initial analysis in order to optimise inter-rater reliability. Additional comparison reviews were carried out across the coding to assure intra-rater and inter-rater reliability. These ongoing reviews allowed for communication between coders to explore alternative interpretations and explanations of the themes and patterns uncovered. Saturation of themes was achieved.

This study was reviewed and approved by an institutional review board in each country in which interviews were done, in addition to all author institutions including Boston Children's Hospital IRB, Kenyatta National Hospital/ University of Nairobi Ethics Review Committee, Research and Ethics Committee of Makerere University College of Health Sciences, Uganda National Council for Science and Technology, Muhimbili University of Health and Allied Sciences Directorate of Research and Publications, and Tanzania Commission for Science and Technology.

\section{RESULTS}

We interviewed 36 individuals as per our protocol. We had a slightly higher representation from senior faculty

${ }^{i}$ Umeå University, Umeå, Sweden. 
Table 1 Participant demographics

\begin{tabular}{llllc}
\hline & Resident & $\begin{array}{l}\text { Junior } \\
\text { faculty }\end{array}$ & $\begin{array}{l}\text { Senior } \\
\text { faculty }\end{array}$ & Total \\
\hline Institution 1 & 3 & 3 & 4 & 10 \\
Institution 2 & 3 & 3 & 3 & 9 \\
Institution 3 & 3 & 2 & 2 & 7 \\
Institution 4 & 3 & 3 & 4 & 10 \\
\hline
\end{tabular}

as we included department chairpersons, and had fewer respondents from one institution due to faculty unavailability (table 1 ).

The participating institutions varied in numbers of guests hosted, ranging from 2 to 10/year, with the global approach to guests appearing to evolve with institutional growth. At larger programmes, senior faculty described hosting many guest faculty early in the institution's existence with lower reliance on guests as the base of local faculty grew. The goals of guest visits also evolved, focusing more on training in specific areas or subspecialties. As programmes grew, some became more selective in the type of guests they were willing to host. There was variation in the degree of effort institutions put into preparing for and hosting guests.

All three subgroups described similar guest experiences, though residents focused more on the benefit of shared knowledge and teaching styles while faculty frequently focused on programmatic aspects.

\section{Themes}

We identified five general themes, many of which were significantly interlinked with subthemes focusing on factors or guest behaviours that influenced the efficacy of guest visits. Emergent themes included:

1. Engagement: the degree to which guest faculty interacted with host faculty and trainees, including time spent and commitment.

2. Understanding of local context: how well guest faculty knew the local context with regard to culture, common diseases, clinical work with limited resources and teaching needs.

3. Meeting the needs of hosts: the degree to which the visit was designed to meet the host's needs.

4. Host and guest resources: the monetary and nonmonetary resources required for visits.

5. Preparation and planning: efforts undertaken by both the guest and host to plan and prepare for the visit.

\section{Benefits}

All 36 respondents indicated some benefit to hosting guest faculty (table 2) even at institutions in which hosting guests was a lower priority. Almost all respondents appreciated sharing of knowledge and approaches to clinical problems.

For smaller institutions with fewer (8 to 10 ) faculty, guest faculty filled gaps by teaching locally unavailable subspecialties and by providing additional faculty to interface with numerous trainees.

Almost all senior faculty and a majority of junior faculty appreciated the guests' ability to help build faculty capacity, predominantly in areas of research and clinical care. A few respondents noted guest faculty involvement in the development of subspecialty fellowship programmes previously unavailable in the region. In several instances, guest exchanges allowed for trainees

Table 2 Quotes on benefits

Position

Our student to faculty ratio is very high, so whenever you have an extra hand, or extra head...it is always something that helps you.

Senior faculty$$
\text { something that helps you. }
$$

Above all, I think our patients, children benefit...You get a second opinion of what might be wrong with

Senior faculty this child.

I believe in Medicine in which we learn not only the science, but also we learn from the person, themselves, the way they conduct themselves..... residents have to choose... "I think this one will be my role model."

Someone can come as a lecturer, but we find that there is an opportunity to do more research here, or... Senior faculty help us in this gap. And back home they have someone who is interested in this area, and he introduces us ...So they [advance the] experience from individual to department to institution.

When the young doctors come here, when they come to Africa, they become activists back in their country... A lot of my friends' perspectives on health have changed because of visiting countries. 
and junior faculty to establish networks that assisted in training and research funding. A majority of senior faculty described guest faculty who were able to expand clinical care capacity through training and materials, such as the ability of one centre to perform peritoneal dialysis. The senior faculty also noted that guests appeared to learn as much as they taught. A few faculty members spoke of the benefits of ongoing relationships with guest faculty, including networking, mentorship and resulting camaraderie.

\section{What was effective and what was challenging?}

Stories about effective guest faculty visits were often mirrored by stories of challenges within the same theme, and subthemes often were identified under multiple, sometimes overlapping, themes (figure 1).

\section{Engagement}

Guest faculty who made longer or repeat visits were viewed as more effective across subgroups (table 3). Short visits were occasionally appropriate for sharing specific desired expertise; however, most respondents described short visits (defined by them as a few days up to 2 weeks) as disruptive and of minimal benefit. Repeat visits were viewed as the most effective.
A majority of faculty members noted challenges of competing visit agendas. Fifteen faculty members described guest faculty interactions that were limited by other academic focuses such as research, and 10 faculty members described guests whose primary goal appeared to be vacation and to 'see Africa'. This subtheme was strongly linked to resources, with many hosts unwilling to request much of guests who were on personal leave and self-funding their visit.

Fourteen respondents preferred the expertise of more senior guests, and some institutions only approved senior faculty visits. However, 11 individuals felt that there was no difference in efficacy of junior versus senior guests. The junior faculty's lack of experience was offset by flexible schedules allowing for longer stays, increased adaptability, fewer competing agendas and increased engagement in clinical teaching.

Many residents and a few faculty members appreciated the collegial learning environment created by some guests, in contrast to the host institution's more hierarchical approach. Similarly, they noted the significant time that guest faculty spent with trainees. Increased engagement led to partnerships characterised by friendship, open communication and trust. While formal evaluations of guest faculty visits were rare unless required by a third party, respondents noted that strong

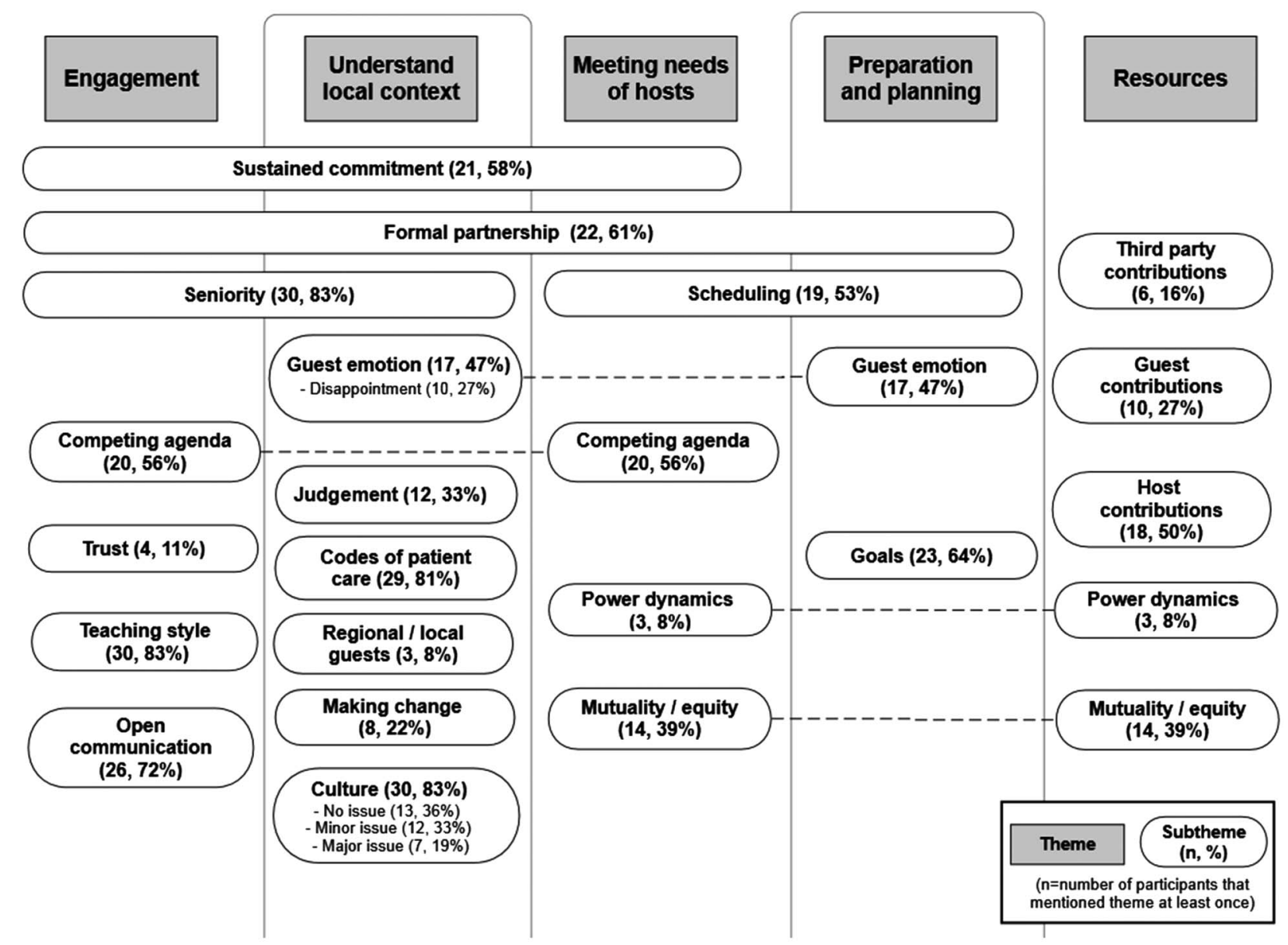

Figure 1 Themes and corresponding subthemes of factors affecting guest faculty effectiveness. 
The ones who have repeated visits.... when they come for the second time, they have seen the setting where we work, they know our needs, and they know exactly what to do and where to go.... But of course, before you come the second time, you must have the first time.

These guys were very student friendly and I think they value student input, and they encouraged student contributions as part of the learning. Because ordinarily in our setting here if I have an answer which I am not sure about I cannot say it because of the repercussion.

They may give the impression that oh they have come to help, but maybe in the background they have come to [tour] because some actually have never come to Africa.

Resident

There are some who have written reports after their visit, and we find their report is really not very good, especially when they are commenting about what their expectations were and what they found.

relationships with guest faculty allowed for reciprocal constructive feedback.

\section{Guest understanding of local context}

Almost all host faculty and residents described frustrations with guests who had minimal experience in limited resource settings, and who lacked pertinent clinical knowledge. Some guests framed discussions about patient management in a way that placed the burden of resource limitations on the host (table 4 ).

Almost half of all respondents across the three subgroups told stories of guest emotional responses to clinical resource limitations. A third of respondents described emotions causing major conflict or resulting in guests leaving early, and a minority of junior faculty and small majority of senior faculty described judgemental responses by guest faculty. A third of the faculty and residents described some guests as 'disappointed', stemming from a lack of clinical resources, the slow pace of change or the guests' own sense of inefficacy.

With the exception of frustration around limited clinical resources, the majority of the respondents reported that cultural differences rarely led to significant conflicts. A third of the respondents indicated that culture was no issue, as either the guests themselves were worldly and well-travelled, or the hosts 'have seen a lot and are quite accommodating'. Another third of the respondents described minor cultural differences which were recognised but did not create much conflict, such as guests' focus on timeliness, direct communication with trainees and patients and informal dress.

Seven respondents described cultural differences that resulted in major conflict, many of which pertained to patient care including when resuscitation was appropriate, disclosing information to paediatric patients or obtaining parental consent. In these stories, host faculty acknowledged having a more 'paternalistic'approach to medical decisions, while guests desired direct engagement with parents and patients. The other major conflicts involved guests' direct and sometimes emotional communication styles when providing feedback, which was perceived by the hosts as 'blunt or rude.'

\section{Meeting needs of hosts}

Scheduling visits appropriately was a concern for a majority of the faculty and residents (table 5). Fitting guests into an ongoing curriculum was challenging, particularly for institutions which relied on guests to teach specific subspecialties. Shared planning of the curriculum worked best, with challenges described when guest faculty did not cover the requested topics. A few faculty members described challenges managing multiple guests from different institutions who visited simultaneously.

Faculty respondents described formal partnerships as most helpful in ensuring that guest faculty visits met the host's needs Within formal partnerships, there was mutual planning for shared goals, clearer expectations on both sides, better evaluation for impact and host faculty maintained greater control over guest visits and resulting teaching.

A majority of the senior faculty spoke of a lack of mutual expectations of host and guest faculty. Several faculty members illustrated this concept with stories of guests who arrived uninvited and expected to work clinically while understanding that would never occur in 
Some of the things take time here, for example, the laboratory investigations. So they would really like to have everything very quick, ... they demand for results, and we don't have them for quite some time, so that becomes a little conflict.

We are overwhelmed by lack of resources.... if you have six children who are in respiratory distress and you have only one port of oxygen what are you going to do?....I think for me what I learnt from them is that one patient is very important... It gives an example to give each patient the maximum amount of concern. In the end you try to...you save those you can.

I remember one of them asking how we didn't have a neonatal intensive care unit and does it bother you that ethically you should be doing this but you're not doing it? So I said okay, of course it bothers me but I faculty don't have the resources to do what I know could be done.

Resident

don't have the resources to do what I know could be done.

And one of them came back and they found that the thing which they recommended at the last visit nothing changed and [one could] really see his disappointment.

Junior

faculty

Maybe in the African culture....People tend to be cautious. They tend to not be so confrontational....They might speak in parables or make suggestions that this might not be the best way to do things, whereas someone from another culture would say, "That's the wrong way to do things". So those kinds of cultural differences in the way people communicate might sometimes create issues.

In training we all speak the same language. So the cultural part, I don't think [cause problems],... the issues that occur are personal issues.

their home institutions. Several host faculty noted a need for more reciprocal faculty exchanges. Only five respondents described having partnerships where trainee or faculty exchange was bilateral.

\section{Resources}

Lack of host resources for guest visits was a substantial challenge noted by almost all faculty members and a minority of residents. Many respondents indicated that they would appreciate having more guest faculty but did not have adequate resources to host them. Guests' provision of time and funding for visits altered the power dynamic such that some hosts felt unable to control the flow of guests (table 6). Even within formal partnerships, host institutions sometimes strained to provide agreed on lodging or transportation.

\section{Preparing for and incorporating guests}

Almost all respondents described collaborative planning efforts as vital to the efficacy of a guest's visit. A majority of the faculty suggested that effective visits require jointly determining the goals, and subsequently deciding appropriate visit length and frequency. Depending on the goals, they could establish a formal partnership which allowed for more accountability, mutuality and equity, and ability to seek third party funding. Partnership development, described by a few senior and junior faculty members, was often a dynamic process beginning with one visit and evolving into more substantial programming as the relationship grew.

Senior host faculty felt they could improve guest efficacy if they appropriately selected guests for expertise and experience. At one institution, multiple senior faculty members described purposely orienting guests and assigning host faculty or administrative contacts to mitigate the guests' potential emotional responses. A few faculty members proposed inviting more regional faculty as guests.

\section{DISCUSSION}

Our resulting themes and stories that were shared point to a struggle of ambivalence, with respondents recognising the advantages of hosting guest faculty, but also identifying situations where it was not beneficial, with hosts having to mitigate associated problems. In some instances, the burden of hosting guests went beyond resources and administration, and involved strained relationships and conferred guilt. We heard many stories of gaps in understanding between guests and hosts. While 
Table 5 Quotes on meeting needs of hosts

Position

We run a semester system and would like to plan for a full semester or a whole academic year. So

Junior faculty knowing who comes out when is important for us,... but if they have fit it in their own personal time back home, it does not always work well for us. So we find sometimes we are trying to adjust things here to accommodate them.

I would say that we prefer the formal partnerships because we can apply some control on how things work and when we have some structure in terms of objectives, then it is possible to evaluate and say this Senior faculty has worked well and this hasn't.

I think as an institution sometimes we give this impression that we are wandering in the dark and we need someone to just tell us what we need, what we want. That is a failing on our part. We need to have

Senior faculty clarity in ourselves, what we need....So the first meeting should leave them with the clear impression that, as an institution, we have our own strategic objectives... We would like certain kinds of help and there is some kind of help we don't want now....Second we need to assert ourselves, so that people do not sort of stampede us into doing something we don't want.

If it's possible to exchange faculty between the two institutions, not always one way, but both ways, so that we learn from each other.

Junior faculty

Table 6 Quotes on resources and preparation

Position

Of course we wanted a particular thing but we are not always in a position to be very exacting in the kind

Senior faculty of person we want. We may be inclined to accept someone who then turns out to be inappropriate.

If it's formal, you plant soil. You plan the time the person should be here and when he/she is needed Senior faculty most, and you plan the curriculum and the topics. So they also come prepared and send someone who is an expert.

I think what makes them effective is ...structure. We need to give them structure, so it's not like they have to make up what they want to give. We need to articulate our needs very clearly. Sometimes we do that Senior faculty by extracting from the curriculum and giving it to them beforehand, and thus communicating with them exactly what we want to achieve and then they are more likely to do the right kind of preparation or they have the right expectations.

the overall themes are reflected in the existing literature on global health and cultural competencies, several new threads emerged that are important to making faculty visits effective and equitable.

\section{Who should be guest faculty?}

Whether guest faculty stayed for years, or gave a single lecture, visits were most valued if the goals were agreed on and tailored to the host institution. Visit duration and guest selection for expertise were dependent on those goals. While wisely recognising that 'before you come the second time, you must have the first time,' faculty struggled to determine which guests might become true collaborators. Institutions most needing guests to 'fill gaps' often relied on guests to fund visits and consequently accepted some visitors who were more of a drain than a benefit. Moving away from a 'beggars cannot be choosers' mentality requires addressing significant resource inequalities to empower host institutions to screen potential guest faculty as some more established programmes already do. More inquiry is needed to determine what degree of stringency is warranted to deter medical tourism and needless diversion of resources, but also allows for initial visits from less experienced guests who may grow to become beneficial partners. 
Our respondents most appreciated guests who could dedicate themselves to building relationships via a sustained commitment. Experience in global settings and adaptability were both prized. While senior guests were acknowledged to have more experience and more knowledge to share, they were often perceived as less available, less adaptable and having more competing agendas than junior guest faculty. Several of our respondents spoke of the desire to select guests with specific expertise that was needed by their institution, which is unsurprising given the lack of paediatric subspecialists in many parts of the globe. Sending and hosting institutions should be mindful of guest selection depending on the goal of the faculty exchange, and potential guests should themselves consider if they are appropriate for the suggested role.

Several respondents noted that the 'guests' were always westerners. Lack of host resources may limit potentially fruitful regional collaborations and desired bilateral exchanges. Literature on ethical partnerships does point to the need for bilateral exchange; however, funding remains a significant barrier, and concurring with our participants' experiences, true bilateral exchange and 'south-south' exchanges remain infrequent. ${ }^{22} 23$

\section{Cultural differences over resource limitations}

We were surprised that two-thirds of the respondents reported that cultural differences were rarely a cause for conflict during faculty visits. Most of the described conflicts involved guest faculty who were emotional, judgemental or aggressively seeking change in response to perceptions of poor quality care. This friction often stemmed from guests' inexperience when confronting true clinical resource limitations. Cultural differences that were anticipated-such as differences in dresswere much better tolerated.

The literature around global health and culture distinguishes between cultural competency, meaning knowledge of different cultures and cultural humility, defined in recent literature as 'having an interpersonal stance ie, other-oriented rather than self-focused, characterised by respect and lack of superiority toward an individual's cultural background and experience'. ${ }^{24-26}$ An inexperienced guest's desire to advocate for each individual patient and improve systems of care may put them in conflict with local providers with a more global view of the community's needs and resources. An approach of cultural humility and learning how to affect change in that specific setting allows for more collaborative approaches to achieve those same goals.

While most global health competencies include domains focused on culture, communication and professionalism, these are challenging areas to assess. ${ }^{18} 1921$ Both sending and hosting institutions need more effective methods to identify guest faculty who may have difficulty adjusting to work in resource-limited settings. More inquiry is needed into effective preparation for global health work, particularly regarding development of adaptability and cultural humility. One novel programme for paediatric residents uses simulation to elicit emotional responses to working with limited clinical resources. ${ }^{27}$ Much of the literature about global health electives encourages mandated preparation for medical trainees and opportunities for debriefing after travelling. ${ }^{28}{ }^{29}$ Such systems are generally less available for faculty members who may have less institutional support for global work.

While evaluation was considered important, our respondents indicated that there were few if any established formal feedback systems. Several respondents mentioned reports written by guests about the host institution following a visit. Most feedback to guests was informal, predicated on either previous formation of strong interpersonal relationships or individual guest faculty seeking feedback from residents. A few third party organisations that arranged faculty exchanges required formal guest evaluations. It is unclear if lack of feedback stemmed from a culture of politeness towards guests, or differentials in access to resources and resulting power imbalances; however, some faculty members did note that joint evaluation was a benefit of formal partnerships. We suggest that a system of feedback to guest faculty should be incorporated into all visits. Such a system would aid hosts in deciding whether to invite a particular guest faculty member for a return visit, and may help guest faculty be more effective on subsequent visits. In the context of partnerships, institutional exchange of feedback may facilitate improved processes in selecting and preparing guest faculty and encourage institutional ownership on the part of sending institutions to address negative feedback with ineffective faculty.

\section{Challenges posed by power imbalances}

Power imbalances are a clearly acknowledged friction in developing effective global health partnerships. ${ }^{13} \quad 30-33$ In a review of partnerships for global health research, Bradley declared that "asymmetry between partners remains the principal obstacle to productive research collaboration." ${ }^{34}$ In 1998, the Swiss Commission for Research Partnerships with Developing Countries (KFPE) proposed 11 principles for research partnerships, and many such as deciding on objectives together, building mutual trust, sharing information and monitoring and evaluating the collaboration were reflected in our findings. ${ }^{35}$ The Canadian Coalition for Global Health Research has developed a partnership assessment tool to assist partners in developing and maintaining equitable and effective partnerships, which also promotes an early focus on developing mutual goals and clear roles for both partners. ${ }^{36}$ While remarkably similar principles for good partnership have been promoted in global health research over the past few decades, our results are evidence that the implementation of these principles is inadequate given the experiences of guest faculty visits as described by our respondents. 
Our results corroborate the need to carefully consider the downstream effects of sourcing funds for faculty visits. ${ }^{36}$ When hosts lacked control over visit resources, the resulting power imbalances limited the hosts' ability to invite guest faculty with desired expertise. This effect was more pronounced for smaller programmes that had a greater need of guest resources. This resource imbalance affected mutuality even in long-term partnerships as evidenced by the lack of desired bilateral exchange. The Medical Education Partnership Initiative, which funded partnerships between medical schools in the USA and Africa, was exemplary in granting host institutions greater control over resources and goals to allow for better alignment of visits with host institutions' needs and more mutual and efficacious partnerships. ${ }^{37}$

A few senior faculty members also remarked on the need for their own programmes to be more directive and specific regarding their institutional goals. Host articulation of a clear strategic plan may allow for better alignment of guests with host goals, promoting more effective visits and less frustration on both sides. While a formal partnership was often viewed as the means to aligned goals, the preferential focus should be the goals of the hosts. The host establishing specific goals even prior to developing a partnership may also make for better institutional partnerships, reduce the number of inoperative memorandums of understanding and allow for joint efforts in pursuing resources to meet those goals, thus further improving balance in power.

\section{Limitations and strengths}

This research was undertaken in East Africa, with guest faculty mostly hailing from the USA, Canada and Western Europe. As such, history and lingering colonial influence may affect both host and guest personal and institutional interactions. While similar influences are most likely present in other low-income countries seeking to expand medical education programmes, the specific context may alter the patterns of interactions noted here. Our inquiry was also specific to paediatric programmes. Further research might explore if these themes hold true for visiting faculty in other subspecialties and settings.

Our author group includes those with experience as guest and host faculty from each of the three participating countries, representing a strength, and also potentially introducing biases. The interviews were carried out by a Kenyan physician researcher. We believe her shared culture in medicine, along with providing confidentiality and anonymity, allowed the respondents to speak freely about their experiences.

\section{CONCLUSION}

Strengthening graduate medical education is a necessary step towards decreasing inequalities in healthcare around the globe. Guest faculty can aid in expanding capacity of paediatric academic centres in low and-middle income countries; however, effective visits require: (1) mutually agreed on goals with appropriate scheduling, visit length and commitment to ensure that the visits meet the host's needs, (2) careful selection and preparation of guest faculty to meet the host's goals, (3) emotional preparation by prospective guests along with host orientation to clinical work in the host's setting, (4) attention to funding sources for the visit and mitigation of resulting power differentials and (5) mutually agreed on methods for bilateral evaluation. Formal partnerships may improve mutuality of faculty exchanges, mitigate inherent power imbalances and allow for more sustained, trusting and open relationships.

\section{Author affiliations}

${ }^{1}$ Division of Medicine Critical Care, Boston Children's Hospital, Boston, Massachusetts, USA

2Division of Infectious Diseases, Boston Children's Hospital, Boston, Massachusetts 02115, USA

${ }^{3}$ Department of Paediatrics, University of Nairobi, Nairobi, Kenya

${ }^{4}$ Department of Paediatrics and Child Health, Muhimbili University of Health and Allied Sciences, Dar es Salaam, Tanzania

${ }^{5}$ Department of Paediatrics, Makerere University, Kampala, Uganda

\section{Handling editor Seye Abimbola}

Acknowledgements The authors would like to thank David N Williams for his invaluable assistance in the design and analysis and reporting of the study, Linda Barnes for training in qualitative interview techniques, and Duncan Maru, Jonathan Borus and Ramnath Subbaraman for their insightful editing.

Contributors CMR substantially contributed to study conception and design, to acquisition, analysis and interpretation of data, drafted the manuscript, critically revised the manuscript and gave final approval for the manuscript to be published. LG and DM substantially contributed to study conception and design, to acquisition, analysis and interpretation of data, critically revised the manuscript and gave final approval for the manuscript to be published. MS substantially contributed to data analysis and interpretation, critically revised the manuscript and gave final approval for the manuscript to be published. EK substantially contributed to study conception and design, critically revised the manuscript and gave final approval for the manuscript to be published. SB-K and AL substantially contributed to study conception and design, to acquisition and interpretation of data, critically revised the manuscript and gave final approval for the manuscript to be published. All the authors agree to be accountable for all aspects of work ensuring integrity and accuracy.

Funding This study was funded by grants from the Global Pediatric Education Consortium and the Boston Children's Hospital Global Pediatrics Program.

Competing interests None declared.

Ethics approval This study was reviewed and approved by Boston Children's Hospital IRB, Kenyatta National Hospital/University of Nairobi Ethics Review Committee, Research and Ethics Committee of Makerere University College of Health Sciences, Uganda National Council for Science and Technology, Muhimbili University of Health and Allied Sciences Directorate of Research and Publications, and Tanzania Commission for Science and Technology.

Provenance and peer review Not commissioned; externally peer reviewed.

Data sharing statement No additional data are available.

Open Access This is an Open Access article distributed in accordance with the Creative Commons Attribution Non Commercial (CC BY-NC 4.0) license, which permits others to distribute, remix, adapt, build upon this work noncommercially, and license their derivative works on different terms, provided the original work is properly cited and the use is non-commercial. See: http:// creativecommons.org/licenses/by-nc/4.0/ 


\section{REFERENCES}

1. Frenk J, Chen L, Bhutta ZA, et al. Health professionals for a new century: transforming education to strengthen health systems in an interdependent world. Lancet 2010;376:1923-58.

2. HEARRT Foundation. Health education and relief through teaching. Secondary Health Education and Relief Through Teaching, 2015. http://www.hearttfoundation.org/ (accessed 30 Nov 2015).

3. Mullan F, Kerry VB. The global health service partnership: teaching for the world. Acad Med 2014;89:1146-8.

4. Binagwaho A, Kyamanywa P, Farmer PE, et al. The human resources for health program in Rwanda-new partnership. $N$ Engl J Med 2013;369:2054-9.

5. Forsyth KD. Training is key to improve child health globally. Lancet 2015;385:327.

6. International Pediatric Academic Leaders Assocation. Secondary. http://www.ipala.org/index.html (accessed 23 Sep 2015).

7. International Pediatric Association. Secondary. 2014 http://www. ipa-world.org/ (accessed 23 Sep 2015).

8. Global Pediatric Education Consortium. Secondary. http://www. globalpediatrics.org/ (accessed 23 Sep 2015)

9. Ganapathi L, Martins Y, Schumann D, et al. Overcoming challenges to develop pediatric postgraduate training programs in low and middle income countries. Educ Health (Abingdon) 2014;27: 277-82.

10. Kraeker C, Chandler C. "We learn from them, they learn from us": global health experiences and host perceptions of visiting health care professionals. Acad Med 2013;88:483-7.

11. Elobu AE, Kintu A, Galukande M, et al. Evaluating international global health collaborations: perspectives from surgery and anesthesia trainees in Uganda. Surgery 2014;155:585-92.

12. Holm JD, Malete M. Nine problems that hinder partnerships in Africa. The Chronicle of Higher Education, 2010.

13. Kolars JC, Cahill K, Donkor P, et al. Perspective: partnering fo medical education in Sub-Saharan Africa: seeking the evidence for effective collaborations. Acad Med 2012;87:216-20.

14. Kasper J, Bajunirwe F. Brain drain in sub-Saharan Africa: contributing factors, potential remedies and the role of academic medical centres. Arch Dis Child 2012;97:973-9.

15. Pust R, Dahlman B, Khwa-Otsyula B, et al. Partnerships creating postgraduate family medicine in Kenya. Fam Med 2006;38:661-6.

16. Olness $\mathrm{K}$, Torjesen $\mathrm{H}$. Use of volunteers to help launch a pediatric residency program in Laos. Ambulatory Child Health 2001;7: 109-17.

17. Azzie G, Bickler S, Farmer D, et al. Partnerships for developing pediatric surgical care in low-income countries. J Pediatr Surg 2008;43:2273-4.

18. Ton TG, Gladding SP, Zunt JR, et al. The development and implementation of a competency-based curriculum for training in global health research. Am J Trop Med Hyg 2015;92:163-71.

19. Jogerst $\mathrm{K}$, Callender $\mathrm{B}$, Adams V, et al. Identifying interprofessional global health competencies for 21 st-century health professionals. Ann Glob Health 2015;81:239-47.
20. Battat R, Seidman G, Chadi N, et al. Global health competencies and approaches in medical education: a literature review. BMC Med Educ 2010;10:94.

21. Rowthorn V, Olsen J. All together now: developing a team skills competency domain for global health education. J Law Med Ethics 2014;42:550-63.

22. Crump JA, Sugarman J, Working Group on Ethics Guidelines for Global Health Training (WEIGHT). Ethics and best practice guidelines for training experiences in global health. Am J Trop Med Hyg 2010;83:1178-82.

23. Miranda JJ, Garcia PJ, Lescano AG, et al. Global health trainingone way street? Am J Trop Med Hyg 2011;84:506; author reply 507.

24. Foster J. Cultural humility and the importance of long-term relationships in international partnerships. J Obstet Gynecol Neonatal Nurs 2009;38:100-7.

25. Tervalon M, Murray-García J. Cultural humility versus cultural competence: a critical distinction in defining physician training outcomes in multicultural education. J Health Care Poor Underserved 1998;9:117-25.

26. Hook JN, Davis DE, Owen J, et al. Cultural humility: measuring openness to culturally diverse clients. J Couns Psychol 2013;60:353-66.

27. Butteris SM, Gladding SP, Eppich W, et al. Simulation Use for Global Away Rotations (SUGAR): preparing residents for emotional challenges abroad-a multicenter study. Acad Pediatr 2014;14:533-41.

28. Balandin S, Lincoln M, Sen R, et al. Twelve tips for effective international clinical placements. Med Teach 2007;29:872-7.

29. Anderson KC, Slatnik MA, Pereira I, et al. Are we there yet? Preparing Canadian medical students for global health electives. Acad Med 2012;87:206-9.

30. Costello A, Zumla A. Moving to research partnerships in developing countries. BMJ 2000;321:827-9.

31. Binka F. Editorial: north-south research collaborations: a move towards a true partnership? Trop Med Int Health 2005;10:207-9.

32. Bleakley A, Brice J, Bligh J. Thinking the post-colonial in medical education. Med Educ 2008;42:266-70.

33. Will tropical medicine move to the tropics? Lancet 1996;347:629.

34. Bradley M. North-South Research Partnerships: Literature Review and Annotated Bibliography. 2006 September, 2006. http://citeseerx. ist.psu.edu/viewdoc/download?doi=10.1.1.543.2630\&rep=rep1\& type=pdf (accessed 8 Jul 2016).

35. Developing SCfRPw, Countries. Guidelines for Research in Partnership with Developing Countries: 11 Principles. 1998. http:// www.kfpe.ch/key_activities/publications/guidelines/guidelines_e.php

36. Afsana K, Habte D, Hatfield J, et al. Partnership Assessment Toolkit 2009 December, 2009. http://www.ccghr.ca/docs/PAT_Interactive_e. pdf (accessed Jul 2015).

37. Olapade-Olaopa EO, Baird S, Kiguli-Malwadde E, et al. Growing partnerships: leveraging the power of collaboration through the Medical Education Partnership Initiative. Acad Med 2014;89 (8 Suppl):S19-23. 